\title{
A Hybrid CFGTSA Based Approach for Scheduling Problem: A Case Study of an Automobile Industry
}

\author{
Felix T. S. Chan, Vikas Kumar, H.K. Chan, S.H. Chung
}

\begin{abstract}
In the global competitive world swift, reliable and cost effective production subject to uncertain situations, through an appropriate management of the available resources, has turned out to be the necessity for surviving in the market. This inspired the development of the more efficient and robust methods to counteract the existing complexities prevailing in the market. The present paper proposes a hybrid CFGTSA algorithm inheriting the salient features of GA, TS, SA, and chaotic theory to solve the complex scheduling problems commonly faced by most of the manufacturing industries. The proposed CFGTSA algorithm has been tested on a scheduling problem of an automobile industry, and its efficacy has been shown by comparing the results with GA, SA, TS, GTS, and hybrid TSA algorithms.
\end{abstract}

Keywords - Scheduling, CFGTSA, GA, SA, TS, chaotic theory.

\section{INTRODUCTION}

$\mathrm{T}$ HE present scenario being a highly competitive one, urges the manufacturers to endeavor hard for achieving the timely and cost effective production, that can facilitate them to respond to the exponentially increasing demands of the customers. All the industries involved in any kind of the production process are required to have an optimal scheduling policy, in order to extract the maximum profit. The scheduling policy, failing to comply with such requirements of enhancing the production, and profit, can cause serious loss to the industries. These requirements of the industries inspired the researchers, to find out an effective way of scheduling the parts/jobs so that they can manage their profits and boost their production. The present paper proposes a Hybrid Chaos-based Fast Genetic Tabu Simulated Annealing (CFGTSA) algorithm to solve the complex scheduling problems, commonly faced by most of the manufacturing enterprises. The proposed algorithm is an integration of the Genetic Algorithm (GA), Tabu Search (TS), and Simulated Annealing (SA), and it uses chaotic sequences generation, instead of the random ones during all

Felix T. S. Chan. is with the Department of Industrial and Manufacturing Systems Engineering, The University of Hong Kong, Pok Fu Lam Road, Hong Kong (phone: 852-2859-7059; fax: 852-2858-6535; e-mail: ftschan@hkucc.hku.hk).

Vikas Kumar is with the Department of Industrial and Manufacturing Systems Engineering, The University of Hong Kong, Pok Fu Lam Road, Hong Kong (e-mail: vikas hku@ yahoo.co.in).

H.K. Chan is with the Department of Industrial and Manufacturing Systems Engineering, The University of Hong Kong, Pok Fu Lam Road, Hong Kong (e-mail: hkchan (a ieee.org).

S.H. Chung is with the Department of Industrial and Manufacturing Systems Engineering, The University of Hong Kong, Pok Fu Lam Road, Hong Kong (e-mail: h9840627@hkusua.hku.hk). the phases of the evolution process.

Genetic Algorithm (GA) [1] is one of the local search techniques that has been of prime interest for the researchers. It has been applied to solve various scheduling problems due to its ability to search the optimal solutions in more convenient manner than the conventional techniques. Murata et al. [2] proposed a multi objective genetic algorithm and applied to flow shop scheduling problem. Resource constrained project scheduling problem was solved by Hartmann [3] using the genetic algorithm with makespan minimization as objective. Genetic algorithm to solve flow shop scheduling problem with parallel machine and special procedure constraint was applied by Wu et al. [4]. In recent years some researchers such as [5], [6], [7], [8], etc. have applied GA to solve the scheduling related problems. However, drawbacks associated with it such as premature convergence, extreme reliance on crossover, and too slow mutation rate reduces its efficiency. Motivated by this later on Kirkpatrick et al. [9] and Creutz [10] proposed a new search technique known as SA to overcome the shortcomings of GA. SA based approach to solve the job shop scheduling problem was attempted by Peter $e t$ al. [11]. Satoh et al. [12] applied SA to solve the power plant generator scheduling problem. Several other scheduling problems have been solved using SA that can be found from the research works of [13], [14], and [15] etc. But even if SA was found more superior than GA, the rate of improvement of solution was very slow; also the computational expensiveness restricted its application in some special cases [10]. TS [16] was also developed aiming reduction in computational time and escaping local minima and, in most cases it outperformed GA and SA. The TS method has also been used by many researchers to solve the scheduling related problems. The applications of the TS in solving the scheduling problems can be found in the works of [17], [18], [19], [20] etc. TS uses a tabu list, which is a short term memory of recently visited solution, in order to escape from local optima but, due to its deterministic nature, it can not avoid cycling. Therefore, it also failed to reduce the computational expensiveness.

Owing to enhanced competence in the market the industries are nowadays searching for an effective method to allocate their jobs on the machines in adequate sequences. They are targeting to reduce their production makespan times, in order to withstand the demand fluctuations, as well as satisfy their customer needs. The random search techniques such as GA, TS, and SA have already found to be efficient in solving the optimization problems. However, changing market scenario demanded a more robust optimization method that can reduce the computational time, as well as converge to the optimal solution in less number of 
iterations. This provoked the researchers to integrate the salient features of evolving search techniques. Glover et al. [21] proposed a hybrid GA and TS algorithm for the optimization problems. Mantawy et al. [22] proposed a hybrid genetic tabu simulated annealing (GTS) algorithm inheriting the GA, TS, and SA methods for solving the unit commitment problem. The hybrid GA algorithm for the open shop scheduling problem was proposed by Liaw [23]. He introduced TS in GA to improve the solution. A hybrid GA algorithm inheriting the famous $N E H$ heuristic has been proposed by Zheng et al. [24] to solve the flow shop scheduling problem. Researchers have recently found that using chaotic sequences, instead of random number generators, significantly improves the result. By using several experimental optimization tests based on the De Jong functions [1], the linear matrix inequality (LMI) Eigen value problems [25], the traveling salesman problem (TSP) [1], and the iterated prisoner's dilemma (IPD) [26], it was shown that convergence can be enhanced using particular chaotic series applied to all variation operators. Caponetto [27] studied the chaotic random generator and showed that it can be applied to evolutionary algorithms to enhance the quality of the solution.

Though, the pressure to reduce computational time and chances to reduce the entrapment of solution, in local optima has, left scope of further research in this area. Inspired by this the authors in this paper have proposed a new hybrid CFGTSA algorithm that integrates the prominent attributes of GA, TS, and SA. The Algorithm also inherits the chaotic sequence based approach to explore the search space in better manner. The algorithm uses the Cauchy probability function, which offers more opportunity to escape from the local minima. The proposed algorithm has been tested on a problem of scheduling the parts in an environment of automobile industry. The detailed description of the problem will be provided in next section. The efficacy of the proposed CFGTSA algorithm has been shown by comparing the results with the GA, SA, TS, GTS, and Hybrid Tabu SA (HTSA) algorithms. The result indicates that the proposed algorithm surpasses other optimization methods.

The paper is organized as follows. Section II discusses the problem environment, objectives, and constraints considered in detailed. The present work considers a production environment of an automobile manufacturing company. Section III gives an overview of the proposed algorithm. The result and discussion has been presented in section IV. Section V concludes the work along with the future suggestions.

\section{PROBLEM DESCRIPTION}

The proposed work considers case study of an automobile industry where scheduling is one of the commonly faced problems. As per the case considered, the parts are required to be scheduled for the three major processes followed in the production plant. The pictorial representations of the processes are shown in Figure 1, and are discussed below:

a) Stamping: In stamping process long rolls of coiled steel are cut into sections (blanks) in the Blanking Line, in preparation to be formed. The forming process is completed when the blanks are automatically inserted into a stamping press between an upper and lower die. The parts formed are then transferred to the welding section, for further assembling them to form the automobile body.

b) Welding: During the welding process, the pieces of

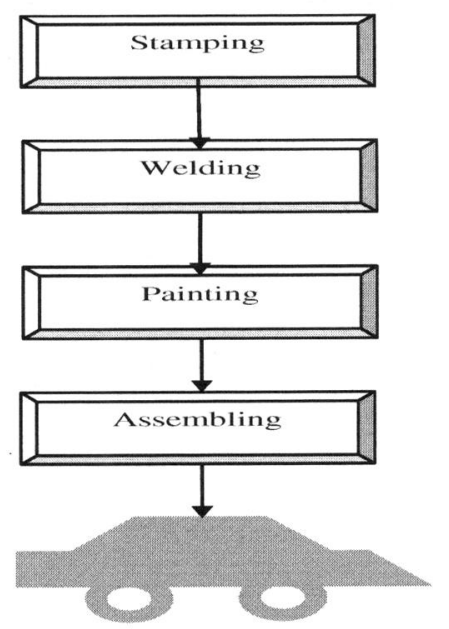

Fig. 1: Production process of an automobile plant.

metal are put together. It is here that the shape of the vehicle comes together. Precision accuracy of these components is required so that all dimensions on the vehicle are within tight specifications. The vehicle assembling takes place in several stages. First underbody and front structure are built. Thereafter, sides and roofs are placed, and finally the closure panels are attached to complete the building process.

c) Painting: In the paint department, coatings are applied to the body to prevent rust and add spectacular color to the vehicle.

d) Assembling: In the general assembly department, all components necessary to finish the vehicle are assembled. Everything from the electrical components to the engine, and tires are put on the vehicles on the conveyor. At the end of this process, the vehicles are ready to be tested before being delivered to consumers.

In the proposed work the emphasis has been given to find an appropriate sequence so that the turbulence in the processing during the stamping, welding, and painting processes can be avoided. Thus, the parts may become available for assembling, leading to the timely and efficient production. The paper aims reduction of the waiting time incurred in between the processes, and reduction of the overall makespan time. Before starting the sequencing of the parts, some of the assumptions can be stated as follows:

(i) All the component parts can be processed for these three operations only in the order of

Stamping $\longrightarrow$ Welding $\longrightarrow$ Painting

(ii) Simultaneously five parts can be assigned at a time.

(iii) The scheduling of the other parts can start only when one of the machines available for stamping is free.

The proposed hybrid CFGTSA algorithm generates a feasible schedule satisfying the concerned objectives. The objective functions considered along with the constraints are discussed in following subsections:

\section{A. Parameters Considered}

$p \quad:$ Part number where, $\mathrm{p}=1,2, \ldots, \mathrm{P}$ 
$o$ : Operation number where, $\mathrm{o}=1,2, \ldots, \mathrm{O}$

$m$ : Machine number where $\mathrm{m}=1,2, \ldots, \mathrm{M}$

$n$ : Number of parts produced where, $\mathrm{n}=1,2, \ldots, \mathrm{N}$

$S_{o p}:$ Starting time of operation o for part $\mathrm{p}$

$C_{o p}:$ Completion time of Operation o for part $\mathrm{p}$

$O P_{n}:$ Total output

$M T_{p}$ : Makespan time for part $\mathrm{p}$ processed on all $\mathrm{M}$

machines

$P T_{p m}$ : Processing time of part $\mathrm{p}$ processed on machine $\mathrm{m}$

$W T_{p m}$ : Waiting time for part $\mathrm{p}$ on machine $\mathrm{m}$

$O T_{n}:$ Total $\mathrm{n}$ number of parts produced

\section{B. Objective Functions}

The scheduling of the rolls of coiled steel plays an important role in automobile industry. Any delay in the process can lead to loss of the production, as the parts may not be available during the assembly stage. Therefore, the reduction of waiting time is of prime concern when enhanced production is targeted. The time up to which the parts are required to wait, until it is conveyed to the next operation is defined as the waiting time. It is expressed as;

Minimize $\{$ Waiting Time $\}=\sum_{m=1}^{M} W T_{p m}$

For any manufacturing enterprise, the product completion time contributes a significant role for faster and reliable deliveries, to comply with customer satisfaction. The shorter makespan time enables the manufacturing enterprises to tackle the demand uncertainty efficiently. The present work focuses on the minimization of the makespan time and it is expressed as;

Minimize $\left\{M T_{p}\right\}=\sum_{m=1}^{M}\left(P T_{p m}+W T_{p m}\right)$

The parts produced are then assembled to construct the final shape of the vehicle. Therefore, the total number of parts manufactured will affect the final assembling process. The proposed work also considers the maximization of the output, i.e. total parts produced satisfying all the constraints. It is expressed as;

Maximize (Output) $=\sum_{n=1}^{N} O T_{n}$

The abovementioned objectives are required to satisfy the constraints that have been described in the next section.

\section{Constraints}

\section{1) Waiting time constraint}

The waiting time has certain restriction, as if exceeds predefined value it may cause disruption in assembling, leading to delayed production. Waiting time is feasible only if;

$$
\sum_{m=1}^{M} W T_{p m} \leq 30
$$

2) Processing Time constraint

This constraint implies that completion time should be either positive or zero.

$$
P T_{p m} \geq 0
$$

3) The parts can't proceed to other operation until the previous operation is completed i.e.,

$$
S_{o p} \succ C_{o p}
$$

4) The parts can't be assigned on more than one machine for the same operation.

5) The machines are subjected to continuous operation while production is in process. Hence, the waiting time within the prescribed limit, during the process should be considered for the maintenance of those machines.

\section{Proposed Algorithm}

The present paper proposes a new hybrid Chaos-based Fast Genetic Tabu Simulated Annealing (CFGTSA) algorithm to solve the scheduling problem prevailing in an automobile industry. The algorithm encapsulates the prominent attributes of GA, TS, and SA optimization methods and uses chaotic sequences [28] during all the phases of evolution process. The proposed algorithm is the extension of the work carried out by Mantawy et al. [22] in which they have proposed GTS algorithm combining the GA, TS, and SA algorithms. The proposed algorithm uses Perturbation methods to explore the neighborhood solution in more efficient manner. Four different types of perturbation methods and a heuristic perturbation method have been used, to explore the search space efficiently. In addition, the proposed algorithm employs the probabilistic transition rule rather than deterministic descent rules. The algorithm replaces the Boltzmann probability function with the Cauchy's probability function during the annealing process of SA.

The steps of the proposed CFGTSA algorithm are described below:

Notations:

$\begin{array}{lll}\mathrm{TP} & : & \text { Transition probability (Cauchy Function) } \\ \Delta \mathrm{F} & : & \text { Change in fitness value } \\ \mathrm{F}\left(\mathrm{Sol}_{\mathrm{p}}\right) & : & \text { Fitness value of the perturbed solution } \\ \mathrm{F}(\mathrm{Sol}) \quad & : & \text { Fitness value of the current solution } \\ \mathrm{TL} & : & \text { Tabu List } \\ \mathrm{T}(\mathrm{k}) & : & \text { Temperature at } \mathrm{k}^{\text {th }} \text { generation } \\ \mathrm{T}_{0} & : & \text { Initial Temperature } \\ \mathrm{k} & : & \begin{array}{l}\text { Number of Iteration in which the temperature is } \\ \text { to be reduced }\end{array} \\ \mathrm{C}_{\mathrm{p}} & : & \text { Control parameter of the cooling schedule } \\ \mathrm{C}_{\mathrm{p}}{ }^{\mathrm{k}} & : & \text { Control parameter at } \mathrm{k}^{\text {th }} \text { number of generation }\end{array}$

STEP 1: Initialize the parameters of GA, TS, and SA i.e., $\mathrm{T}=\mathrm{T}_{0}=500^{\circ} \mathrm{C}, \mathrm{TL}=0, \mathrm{Cp}=\mathrm{Cp}^{\mathrm{k}}$, Reject $=0$.

STEP 2: Generate initial population by generating a set of feasible solutions

STEP 3: Evaluate the fitness value of each population

STEP 4: Generate a new solution from the existing solution. If the perturbated solution is a feasible one then move to next step otherwise regenerate the solution till feasible solution is obtained.

STEP 5: Apply GA parameters to generate a new set of populations as follows;

- The best solution is added to new population from the current population

- Generate new neighbor members in the new population using TS algorithm. 
- Apply the crossover operator (Single point crossover and chaotic sequences are used for the choice of points) to complete the members of the new population.

- Apply the mutation operator (swap mutation and chaotic sequences are used for choosing the positions of chromosomes) to the new population.

STEP 6: Compute $\Delta \mathrm{F}=\mathrm{F}\left(\mathrm{Sol}_{p}\right)-\mathrm{F}(\mathrm{Sol})$. If $\Delta \mathrm{F} \geq 0$ then go to STEP 7 else, go to STEP 9.

STEP 7: Assign $\mathrm{Sol}=\mathrm{Sol}_{\mathrm{p}}$, Reject $=0$, Go to STEP 9

STEP 8: Compute the Transition Probability (TP) and generate random number $\mathrm{R}$ between $(0,1)$. If $(\mathrm{TP} \leq$ R) then go to STEP 10 else go to next STEP 9.

STEP 9: Assign Sol $=\mathrm{Sol}_{p}$. Include $\mathrm{Sol}_{\mathrm{p}}$ in the Tabu list. A $\mathrm{F}\left(\mathrm{Sol}_{\mathrm{p}}\right)$

STEP 10: Reject $=$ Reject +1 ; If Reject $\geq 3$ go to STEP 14 else go to STEP 11

STEP 11: Calculate the new temperature of SA algorithm cooling schedule

- Calculate the new temperature $C_{p}{ }^{k}=C_{p}(\beta)^{k}$, where $0<\beta<1$

STEP 12: Apply SA algorithm to test the members of the new population.

STEP 13: Make the current population to be the new population

STEP 14: Freeze, if the stopping criterion is satisfied. The solution is the optimal/near optimal.

The detailed description of the steps of the proposed algorithm has been discussed below:

A. Solution Encoding

The proposed CFGTSA algorithm uses the operation oriented encoding scheme for the ease of calculation. The first row represents the part number where as the further rows represent the machines where the subsequent three operations are to be performed. Sample representation of the encoding for 10 parts and 5 machines is shown below;

$\begin{array}{llllllllll}6 & 2 & 9 & 7 & 4 & 1 & 5 & 3 & 8 & 10 \\ 1 & 4 & 3 & 3 & 2 & 5 & 4 & 1 & 5 & 2 \\ 5 & 4 & 4 & 2 & 1 & 2 & 3 & 1 & 5 & 1 \\ 2 & 4 & 3 & 1 & 3 & 2 & 5 & 4 & 2 & 5\end{array}$

\section{B. Initialization}

In the present work initial solution is randomly generated as it is believed that randomly generated solution initiates a more effective search thereafter, the chaotic sequences are followed for solution generation.

\section{Neighborhood generation (Perturbation method)}

Perturbation controls the searching capacity and convergence trend of the algorithm. In the proposed work four perturbation methods [27], [28] Logistic Map Based heuristic perturbation method, Tent Map Based heuristic perturbation method, Sinusoidal Integrator Based heuristic perturbation method, and Gauss Map Based heuristic perturbation method, along with a hybrid perturbation method, known as Chaotic Function Based Heuristic perturbation method have been used. These perturbation methods make use of Heuristic Based perturbation method, where, $\mathrm{N}$ bits of initial solution is selected randomly and thereafter all possible permutation of selected bits is generated. Finally the best one is selected as final solution for further calculation. The procedure of the new hybrid perturbation is described below:
Step 1: Randomly select a chaotic sequence strategy among aforementioned five strategies.

Step 2: Perform Step 1 to Step 3 as mentioned in Logistic Map Based Heuristic Perturbation Method stated below;

$>$ Logistic Map Based Heuristic Perturbation Method ( $L M H P M)$

It is one of the simplest dynamic systems supporting chaotic behavior. The Logistic Map equation is expressed as follows.

$$
X_{k+1}=\mu X_{k}\left(1-X_{k}\right)
$$

Where, $\mu$ is tuning parameter. In the proposed work $\mathrm{X}_{0}=0.2027$ and $\mu=4$.

$$
\mathrm{N}_{\mathrm{k}+1}=\mathrm{I} \times \mathrm{X}_{\mathrm{k}+1}
$$

Where, $\mathrm{I}=$ number of bits in the solution.

Step 1: Pick up $\mathrm{N}$ different bits (from the set of random number generated by the selected chaotic sequence strategy).

Step 2: From the exiting alternative, change the operating machine corresponding to selected operations in the step 1 .

Step 3: Considering all possible permutation of first row of selected bits, select the best neighborhood for further calculation

\section{Probabilistic transition rule}

In this algorithm, the Cauchy function (TP) has been used. The next inferior iteration solution is selected if, TP $>\mathrm{R}$ where $\mathrm{R}$ is a random number. For each perturbed solution, inferior in comparison with candidate solution; the transition probability can be calculated as;

$$
T P=\frac{T(k)}{T^{2}(k)+(\Delta F)^{2}}
$$

Where, $\mathrm{T}(\mathrm{k})=$ annealing temperature at the $\mathrm{k}^{\text {th }}$ Iteration.

$$
\Delta F=F(\text { Sol })-F\left(\text { Sol }_{p}\right)
$$

\section{E. Annealing schedule}

The following annealing schedule is followed:

$$
T(k)=\frac{T_{0}}{1+\ln k}
$$

Where, $T_{0}=$ Initial temp.

\section{F. Tabu list}

Tabu list is used to check whether the pretreated solution is again visited or not during the each step of algorithm, and thus helps in restricting the algorithm to revisit the pre-visited solutions [21].

\section{G. Aspiration}

This tabu search variable denoted by ' $A$ ' restricts the search from being trapped in a solution, surrounded by the tabu neighbors, by choosing the solution whose objective function is greater for further exploration.

\section{H. Reject}

The pretreated solutions are often rejected on the basis of probability consideration. After a certain limit of rejection, it is assumed that there is no superior solution existing in the neighborhood, and the search has reached a near optimal / optimal solution.

\section{Stopping criteria}

In order to stop the searching procedure from roaming into the solution space, following stopping criteria has been adopted;

\section{a. Number of iterations}

During experimentation, it was found that as the number of iteration increases, equivalent temperature falls to a 
minimum level. Any further reduction in temperature would not be useful, because at low temperature the possibility of accepting inferior solution is very small, and results obtained are virtually indistinguishable from the optimal solution

\section{b. Variable reject}

When reject counter attains predetermined fixed value then it means that, no optimal or near optimal solution has been visited during the last few steps. Therefore, the probability of obtaining better solution is small and hence, the searching procedure is stopped.

\section{RESUlT AND DISCUSSION}

In the present work the efficacy of the Hybrid CFGTSA algorithm has been shown by solving a considered test problem of an automobile production plant environment, and comparing the results with GA, TS, SA, GTS, and HTSA algorithms. There are total 20 parts that are to be manufactured by passing through three major operations to form the vehicle body. There are 5 machines for each operation thus; there are total 15 machines for three operations. The parts are subjected for processing on the alternative machines available to them while passing through these three major operations but, each part doesn't

TABLE I

PARTS ALTERNATIVES AND PROCESSING TIME CORRESPONDING TO THE OPERATIONS

\begin{tabular}{|c|c|c|c|c|c|c|}
\hline \multirow{2}{*}{$\begin{array}{l}\text { Parts } \\
\text { No. }\end{array}$} & \multicolumn{2}{|c|}{ Stamping } & \multicolumn{2}{|c|}{ Welding } & \multicolumn{2}{|c|}{ Painting } \\
\hline & $\mathrm{M} / \mathrm{C}$ & PT & $\mathrm{M} / \mathrm{C}$ & PT & $\mathrm{M} / \mathrm{C}$ & PT \\
\hline \multirow[t]{2}{*}{1} & M12 & 30 & M22 & 15 & M35 & 30 \\
\hline & M15 & 30 & M24 & 30 & & \\
\hline \multirow[t]{2}{*}{2} & M12 & 30 & M25 & 15 & M32 & 30 \\
\hline & M14 & 30 & & & M33 & 30 \\
\hline \multirow[t]{2}{*}{3} & M11 & 45 & M22 & 30 & M34 & 45 \\
\hline & M15 & 45 & M23 & 15 & M35 & 45 \\
\hline \multirow[t]{2}{*}{4} & M12 & 30 & M21 & 30 & M31 & 30 \\
\hline & M13 & 45 & & & M35 & 45 \\
\hline \multirow[t]{2}{*}{5} & M11 & 30 & M22 & 30 & M32 & 30 \\
\hline & M15 & 30 & M24 & 15 & M35 & 30 \\
\hline \multirow[t]{2}{*}{6} & M12 & 30 & M23 & 30 & M33 & 45 \\
\hline & M13 & 45 & M25 & 30 & M34 & 30 \\
\hline \multirow[t]{2}{*}{7} & M14 & 30 & M23 & 15 & M31 & 45 \\
\hline & & & M25 & 30 & M34 & 45 \\
\hline \multirow[t]{2}{*}{8} & M11 & 45 & M21 & 30 & M35 & 30 \\
\hline & M15 & 30 & M24 & 15 & & \\
\hline \multirow[t]{2}{*}{9} & M12 & 30 & M22 & 15 & M31 & 45 \\
\hline & M15 & 30 & M23 & 15 & M32 & 30 \\
\hline \multirow[t]{2}{*}{10} & M11 & 30 & M21 & 30 & M32 & 30 \\
\hline & M14 & 30 & M25 & 15 & M35 & 30 \\
\hline \multirow[t]{2}{*}{11} & M13 & 45 & M21 & 30 & M31 & 45 \\
\hline & M14 & 45 & M24 & 30 & M34 & 30 \\
\hline \multirow[t]{2}{*}{12} & M11 & 30 & M22 & 15 & M33 & 30 \\
\hline & M12 & 30 & M25 & 30 & M35 & 30 \\
\hline \multirow[t]{2}{*}{13} & M14 & 30 & M23 & 15 & M32 & 30 \\
\hline & M15 & 30 & M24 & 30 & M33 & 45 \\
\hline \multirow[t]{2}{*}{14} & M11 & 45 & M21 & 30 & M31 & 30 \\
\hline & M13 & 30 & M24 & 30 & & \\
\hline 15 & M12 & 30 & M23 & 30 & M34 & 30 \\
\hline \multirow[t]{2}{*}{16} & M13 & 30 & M22 & 30 & M33 & 30 \\
\hline & & & & & M35 & 30 \\
\hline \multirow[t]{2}{*}{17} & M11 & 30 & M25 & 15 & M32 & 30 \\
\hline & M14 & 45 & & & M33 & 30 \\
\hline \multirow[t]{2}{*}{18} & M11 & 30 & M23 & 30 & M34 & 30 \\
\hline & M12 & 30 & M25 & 30 & & \\
\hline \multirow[t]{2}{*}{19} & M13 & 30 & M21 & 30 & M31 & 30 \\
\hline & & & M24 & 30 & M34 & 45 \\
\hline \multirow[t]{2}{*}{20} & M14 & 30 & M21 & 30 & M33 & 30 \\
\hline & M15 & 45 & M24 & 15 & M35 & 30 \\
\hline
\end{tabular}

have access to all the alternatives, due to the capacity limitation of the machines, and the shape, and size of the parts to be produced. The alternatives available for the parts, as well as their corresponding processing times are shown in Table I. The aim of the proposed work is to minimize the waiting time, and makespan time, as well as maximize the output by proper scheduling of the parts.

The comparative result of the undertaken problem has been shown in Table II. The Gantt chart of the optimal

TABLE II

COMPARATIVE COMPUTATIONAL RESULTS

\begin{tabular}{ccccccc}
\hline $\begin{array}{c}\text { Scheduling } \\
\text { Methods }\end{array}$ & GA & SA & TS & $\begin{array}{c}\text { HTS } \\
\text { A }\end{array}$ & GTSA & CFGTSA \\
\hline $\begin{array}{c}\text { No. of } \\
\text { Iterations }\end{array}$ & 640 & 780 & 610 & 632 & 605 & 584 \\
$\begin{array}{c}\text { CPU time } \\
\text { Waiting }\end{array}$ & 15 & 21 & 16 & 11 & 9 & 7 \\
$\quad \begin{array}{c}\text { Time } \\
\text { Makespan } \\
\text { Time }\end{array}$ & 225 & 225 & 225 & 210 & 205 & 195 \\
Output & 17 & 18 & 19 & 20 & 20 & 20 \\
\hline
\end{tabular}

schedule obtained by CFGTSA has been shown in Figure 2. The comparative convergence plot has been presented in

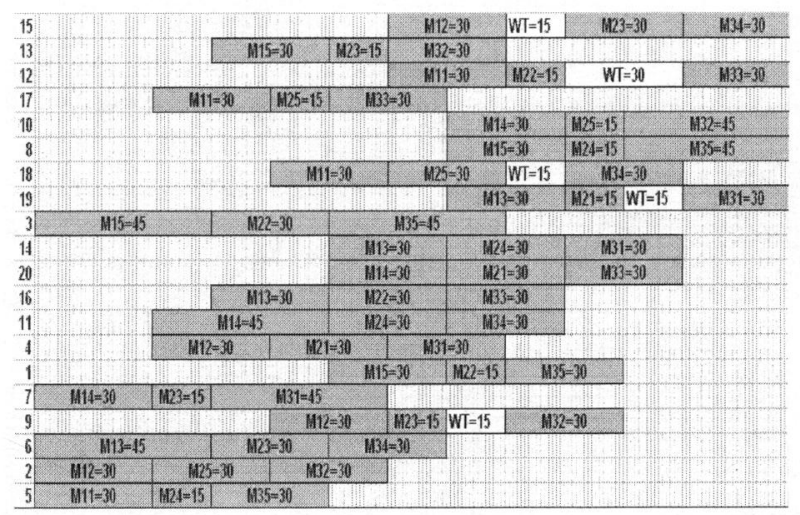

Fig. 2: Optimal seauence obtained bv CFGTSA algorithm

Figure 3. The result shows that the proposed CFGTSA algorithm surpasses other optimization methods such as GA, SA, TS, GTS, and HTSA algorithms. The crossover and mutation ratio was considered to be 0.5 and 0.01

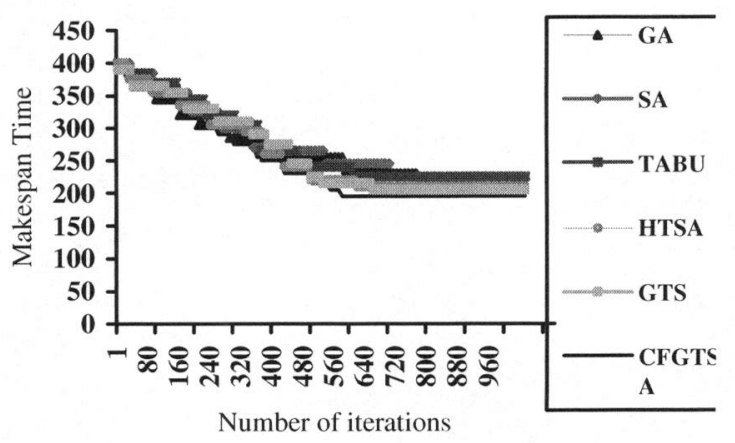

Fig. 3: Comparative convergence plot with CFGTSA

respectively in GA. The initial temperature was assigned to be $300^{\circ} \mathrm{C}$ and $500^{\circ} \mathrm{C}$ in SA and HTSA respectively. The waiting time has been reduced to a great extent as compared to other methods. Similar improvements can be visualized in makespan time reduction, and output maximization. In terms of the computational time too, the proposed CFGTSA algorithm outperforms the other optimization methods, and 
emerges as the best optimization technique. These assessments show the efficacy and robustness of the CFGTSA algorithm in solving the complex scheduling problems. The proposed algorithm has been coded in $\mathrm{C}++$ and tested on Pentium IV $2.2 \mathrm{GHz}$ processor.

\section{CONCLUSION}

The present paper proposes a Hybrid Chaos-based Fast Genetic Tabu Simulated Annealing (CFGTSA) algorithm to solve the scheduling problem of an automobile industry. Enticed by the salient features of the GA, TS, and SA, the authors have integrated them along with the chaotic sequencing to find an appropriate schedule, so that the total waiting time and overall makespan time can be reduced, in order to facilitate smooth production. The paper also aims in maximization of the output in order to fulfill the customer demand and gain more profits. The proposed algorithm has been compared with the results those obtained by the GA, SA, TS, GTS, and Hybrid TSA algorithms. The results showed that CFGTSA algorithm out performed the other methods. The algorithm combining the three search heuristics encapsulates the prominent features from each of them, and does away with their individual shortcomings. The chaotic sequencing enhances the quality of the solution.

The proposed algorithm possesses prominent aspect of its implementation in solving the other complex combinatorial optimization problems. The proposed algorithm can be tested to solve more complex multi objective real time problems. The future work needs to be carried to enhance the precision of the algorithm by modifying the selection technique of crossover and mutation rate. Nowadays the fuzzy logic controllers (FLC) [29], [30] are being used to reduce the chances in local optima, as well as reduce the computational time. The fuzzy controller can be introduced to regularly update the crossover and mutation ratio which can reduce the computational time to a great extent. The implementation of FLC can enhance the precision of the algorithm to converge to the optimal solution in less computational time, and algorithm can become more robust and efficient in handling the uncertainties of demand. Therefore, further work should be focused on the implementation of the CFGTSA algorithm in diverse field of manufacturing related problems with enhanced precision.

\section{REFERENCES}

[1] D. E. Goldberg, Genetic Algorithm in Search Optimization and Machine Learning. Reading, MA: Addison-Wesley, 1989.

[2] T. Murata., H. Ishibuchi, and H. Tanaka., Multi-objective genetic algorithm and its applications to flows hop scheduling, Computers and Industrial Engineering, 1996, Vol. 30, No. 4, pp. 957-968.

[3] S. Hartmann, A competitive genetic algorithm for resource-constrained project scheduling, Naval Research Logistics 1998, Vol. 45, No. 7, pp. $733-750$

[4] Y. Wu, M. Liu, and C. Wu, A genetic algorithm for solving flow shop scheduling problems with parallel machine and special procedure constraints, Machine Learning and Cybernetics, 2003 International Conference on Publication, 2003, Vol. 3, pp. 17741779

[5] M. K. Tiwari, and N. K. Vidyarthi, Solving machine loading problems in a flexible manufacturing system using a genetic algorithm based heuristic approach, International Journal of Production Research, 2000, Vol. 38, No. 14, pp. 3357 - 3384

[6] A. H. Lee and S. S. Kim, Integration of Process Planning and Scheduling Using Simulation Based Genetic Algorithms,
International Journal of Advanced Manufacturing Technology, 2001, Vol. 18, No. 8, pp. $586-590$.

[7] A. Turkcan, and M. S. Akturk, A problem space genetic algorithm in multi objective optimization, Journal of Intelligent Manufacturing, 2003, Vol. 14, No. 3-4, pp. 363 - 378.

[8] F. T. S. Chan, S. H. Chung, and P.L.Y. Chan, An Introduction of Dominant Genes in Genetic Algorithm for Scheduling of FMS, Proceedings of the 2005 IEEE International Symposium on Intelligent Control, Limassol, Cyprus, 2005, June 27-29,.pp. 1429-1434.

[9] S. Kirkpatrick, C. D. Gelatt, and M.P. Vecchi, Optimization by simulated annealing. SCIENCE, 1983, Vol. 220, pp. 671-680.

[10] M. Creutz, Micro canonical Monte Carlo Simulation. Phys. Rev. Lett., 1983, Vol. 50, No. 19, 1411-1414.

[11] J. M. Peter, V. Larrhoven, E. H. L. Aarts, and J. K. Lenstra, Job Shop Scheduling by Simulated Annealing, Operations Research, 1992, Vol. 40, No. 1, pp. 113-125

[12] T. Satoh, and K. Nara, Maintenance scheduling by using simulated annealing method (power plant), IEEE Transactions on Power Systems, 1991, Vol. 6, No. 2, pp. 850-857

[13] O. Catoni, Solving Scheduling Problems by Simulated Annealing, Society for Industrial and Applied Mathematics, 1998, Vol. 36, No. 5, pp. 1539-1575.

[14] K. Krishna, K. Ganeshan, and D. J. Ram, Distributed simulated annealing algorithms for job shop scheduling, IEEE Transactions on Systems, Man and Cybernetics, 1995, Vol. 25, No. 7, pp. 1102-1109

[15] S. Mishra, M. K. Tiwari, and R. S. Lashkari, A fuzzy goal-programming model of machine-tool selection and operation allocation problem in FMS: a quick converging simulated annealing-based approach, International Journal of Production Research, 2006, Vol. 44, No. 1, pp. 43 - 76

[16] F. Glover, Tabu Search-Part I, ORSA Journal on Computing, 1989 , Vol. 1, No. 3, 190-206.

[17] M. Dell'Amico and M. Trubian, Applying Tabu Search to the job-shop scheduling problem, Annals of Operations Research, 1993, Vol. 41, No. 3, pp. $231-252$.

[18] B. Xaiomin, and S. M Shahidehpour, Hydro-thermal scheduling by tabu search and decomposition method, IEEE Transactions on Power Systems, 1996, Vol. 11, No. 2, pp. 968-974.

[19] M. A. Reed, V. A. Armentano, and D. P. Ronconi, Tabu search for total tardiness minimization in flow shop scheduling problems, Computers and Operations Research, Vol. 26, No. 3, 1999, pp. 219-235(17)

[20] R. L. Daniels and J. B. Mazzola, A tabu-search heuristic for the flexible-resource flow shop scheduling problem, Annals of Operations Research, 1993, Vol. 41, No. 3, pp. $207-230$

[21] F. Glover, J. P. Kelly, and M. Laguna, Genetic algorithms and tabu search: hybrids for optimization, Computers and Operations Research, 1995, Vol. 22, No. 1, pp. $111-134$

[22] A. H. Mantawy, Y. L. Abdel-Magid, and S. Z. Selim, Integrating genetic algorithms, tabu search, and simulated annealing for the unit commitment problem, IEEE Transactions on Power Systems, 1999, Vol. 14, No. 3, pp. 829-836.

[23] C. F. Liaw, A hybrid genetic algorithm for the open shop scheduling problem, European Journal of Operational Research, 2000, No. 124 , pp. $28-42$

[24] D. Z. Zheng, and L. Wang, An Effective Hybrid Heuristic for Flow Shop Scheduling, The International Journal of Advanced Manufacturing Technology, 2003, Vol. 21, No. 1, pp. $38-44$.

[25] S. Boyd, L. E. Ghaoui, E. Feron, and V. Balakrishanm, Linear Matrix Inequalities in System and Control Theory. Philadelphia, PA: SIAM Books, 1994.

[26] D. Fogel, Evolutionary Computation. Piscataway, NJ: IEEE Press, 1995.

[27] R. Caponetto, Chaotic Sequences to Improve the Performance of Evolutionary Algorithms, IEEE Transactions on Evolutionary Computation, Vol. 7, No. 3, 2003, pp. 289-304.

[28] T. S. Parker, and L. O. Chua, Practical Numerical Algorithms for Chaotic System. Berlin, Germany: Springer-Verlag, 1989

[29] K. W. Kim, M. Gen, and G. Yamazaki, "Hybrid genetic algorithm with fuzzy logic for resource-constrained project scheduling", Applied Soft Computing, 2003, 2/3F, pp. 174-188.

[30] F. T. S. Chan, V. Kumar, and M.K. Tiwari, Optimizing the Performance of an Integrated Process Planning and Scheduling Problem: An AIS-FLC based approach, IEEE CIS-RAM 06 Conference., Thailand, 2006 